\title{
EVALUACIÓN DEL ESTRÉS TÉRMICO DURANTE EL VERANO 2015 EN LOS AMBIENTES DE UNA FÁBRICA DE CHOCOLATES EN LA CIUDAD DE LIMA
}

\section{EVALUATION OF HEAT STRESS DURING THE SUMMER 2015 IN CHOCOLATE FACTORY ENVIRONMENTS IN THE CITY OF LIMA}

\author{
${ }^{1}$ Julio Alfonso Arakaki Kiyán, ${ }^{2}$ Oscar Enrique Tang Cruz y ${ }^{3}$ Roxani Yaringaño Limache
}

\begin{abstract}
Resumen
El presente trabajo de investigación tuvo como objetivo evaluar el riesgo de estrés térmico en trabajadores de una planta de fabricación de chocolate ubicada en la ciudad de Lima durante la época de verano, usando el método denominado Índice WBGT de acuerdo a la norma NTP RM 375 -2008 y la guía 2 del DS 055-1010 evaluándose paralelamente el gasto metabólico de cada trabajador. La evaluación fue efectuada en 2 etapas del proceso de fabricación en caliente denominados Tostado - Secado y Confitado Caliente. El equipamiento usado consto de un equipo de medición de estrés térmico marca EXTECH. Los resultados de las mediciones nos indican que los valores de WBGT en las dos etapas del proceso están por encima a los establecidos por la norma, tanto para los valores de acción como para los límites máximos permisibles.
\end{abstract}

Palabras claves: estrés térmico, gasto metabólico, temperatura de globo, índice WBGT.

\begin{abstract}
This research aimed to evaluate the risk of heat stress in workers at chocolate manufacturing plant. The present study aimed to evaluate the thermal stress levels in the chocolate manufacturing plant located in the city of Lima during the summer, using a method called WBGT index according to the NTP RM 375 -2008 and the Guia 2 of the DS 055-2010 . The evaluation was carried out in two stages of the manufacturing process called roasted - drying and hot confit. The equipment used were measuring equipment EXTECH brand for thermal stress. The main conclusion was that the measurements indicated that the WBGT values in the two stages of the process are above the standard set by both the action values and for the maximum permissible limits.
\end{abstract}

Key words: Heat stress, metabolic rate, globe temperature, WBGT index.

\section{Introducción}

En la actualidad la industria se ha desarrollado rápidamente en nuestro país lo que ha conllevado también a que los trabajadores reclamen por las condiciones ambientales dentro del trabajo, en especial las relacionadas a las altas temperaturas del aire, fuentes de calor radiante, alta humedad y contacto con fuentes calientes, en algunas con ambientes cerrados como las industrias de fundición, plantas de cerámicas, fábricas de vidrio, plásticos, panaderías, industrias y minería, y en otros en ambientes externos a cielo abierto, como la construcción, reciclaje en climas cálidos, afectan la salud y la eficiencia de los trabajadores. En los últimos años en el Perú se han venido dando normas que permiten regular las exposiciones a las altas temperaturas como la Norma Básica de Ergonomía y de Procedimiento de Riesgo Disergonómico (NTP RM - 375-2008-TR), Reglamento de Seguridad y Salud Ocupacional y otras medidas complementarias en Minería (DS 055-2010MEM -2010) Guía 2; DS 42-F de Seguridad Industrial y la norma RM 318-2010. Además de las normas internacionales ISO 7243-1982 Hot EnvironmentsEstimation of the heat stress on working man based on WBGT index (wet bulb and globe temperature): y las norma NTP 18 de España.

El objetivo principal del presente trabajo es evaluar el nivel de estrés térmico durante la época de verano en dos procesos en caliente en la industria de chocolate como son, Tostado - Secado y Confitado Caliente, en una planta ubicada en la ciudad de Lima; para lo cual, se ha utilizado el método de índice WBGTi, descrito en la metodología.

\footnotetext{
${ }^{1}$ Universidad Nacional Agraria La Molina, Lima, Perú. E-mail: jarakaki@lamolina.edu.pe

${ }^{2}$ Universidad Nacional Agraria La Molina

${ }^{3}$ Universidad Nacional Agraria La Molina.
} 


\section{Materiales y Métodos}

Los gastos metabólicos fueron estimados de acuerdo a las distintas actividades efectuadas por los trabajadores y en base las normas existentes. RM 375 -2008 y la Guía 2 del DS 0552010 y el tipo de trabajo de acuerdo a la ACGIH 2007. Tabla 1

Tabla 1. Categorías de gastos metabólicos para una persona de $70 \mathrm{~kg}$.

\begin{tabular}{lcc}
\hline Categoría & $\begin{array}{c}\text { Gastos metabólicos } \\
\text { promedio } \\
(\mathrm{W})\end{array}$ & $\begin{array}{c}\text { Rango metabólico } \\
\text { (W) }\end{array}$ \\
\hline Descanso & 115 & - \\
Ligero & 180 & $115-235$ \\
Moderado & 300 & $235-360$ \\
Pesado & 415 & $360-470$ \\
Muy & 520 & - \\
Pesado & &
\end{tabular}

Para la evaluación del Estrés Térmico en la planta se usará el método WBGTi (para ambientes interiores) Temperatura de bulbo húmedo y temperatura de globo en ambientes interiores, de acuerdo con la ecuación:

$$
W B G T i=T B H \times 0.7+T G \times 0.5
$$

Donde: TBH: temperatura de bulbo húmedo en ${ }^{\circ} \mathrm{C}$,

TG temperatura de globo en ${ }^{\circ} \mathrm{C}$

Las mediciones de los valores de WBGTi serán obtenidos con el equipo EXTECH TH30.

Los valores límites máximos permisibles de comparación están dadas por la norma RM 375- 2008 - la Norma ISO 7247 tabla 2.

Equipos: Un equipo de estrés térmico marca EXTECH. Modelo: TH30. Heat Stress Index mide el calor que se siente cuando la humedad es combinada con la temperatura, el movimiento del aire, y el calor radiante.

Tabla 2. Valores límites máximos permisibles para el índice WBGTi de acuerdo con la categoría de trabajo y el porcentaje de trabajo descanso.

\begin{tabular}{ccccccccc}
\hline Tipo de Trabajo & $\begin{array}{c}\text { Liviano } \\
\text { Aclima. }\end{array}$ & $\begin{array}{c}\text { Moderado } \\
\text { Aclima. }\end{array}$ & $\begin{array}{c}\text { Pesado } \\
\text { Aclim. }\end{array}$ & $\begin{array}{c}\text { Muy } \\
\text { Pesado } \\
\text { Aclim. }\end{array}$ & $\begin{array}{c}\text { Liviano } \\
\text { No Aclima. }\end{array}$ & $\begin{array}{c}\text { Moderado No Aclima. } \\
\text { No Aclim. }\end{array}$ & $\begin{array}{c}\text { Pesado } \\
\text { Nosado } \\
\text { No Acli. }\end{array}$ \\
\hline 100\%Tra. 0\%Des. & 29.5 & 27.5 & 26.0 & ---- & 27.5 & 25.0 & 22.5 & ---- \\
75\%Tra. 25\%Des. & 30.5 & 28.5 & 27.8 & ---- & 29.0 & 26.5 & 24.5 & ---- \\
50\%Tra. 50\%Des. & 31.5 & 29.5 & 28.5 & 27.5 & 30.0 & 28.0 & 26.5 & 25.0 \\
25\%Tra. 75\%Des. & 32.5 & 31.0 & 30.0 & 29.5 & 31.0 & 29.0 & 28.0 & 26.5 \\
\hline
\end{tabular}

Fuente: RM 375:-2008 TR

De otro lado, la ACGIH 2007 recomienda evaluar los índices de WBGTi de acuerdo a un límite de acción, que podría considerarse como los valores recomendados de trabajo; valores inferiores a los LMP, como se muestra en la tabla 3

De acuerdo con Iñaki 2000?, debe medirse además la temperatura del bulbo seco, bulbo húmedo, temperatura de globo y humedad relativa.
Tabla 3. Valores limites de acción para los WBGTi de trabajo recomendados por la ACGIH y la Guia 2 del DS 055-2010.

\begin{tabular}{ccccc}
\hline Tipo de Trabajo & $\begin{array}{c}\text { Liviano } \\
\text { Aclima. }\end{array}$ & $\begin{array}{c}\text { Moderado } \\
\text { Aclima. }\end{array}$ & $\begin{array}{c}\text { Pesado } \\
\text { Aclima. }\end{array}$ & $\begin{array}{c}\text { Muy Pesado } \\
\text { Aclima. }\end{array}$ \\
\hline $\begin{array}{c}\text { 75 a } 100 \% \\
\text { Trabajo }\end{array}$ & 28.0 & 25.0 & - & - \\
50 a75 \% Trabajo & 28.5 & 26.0 & 24.0 & - \\
25 a 50 \% Trabajo & 29.5 & 27.0 & 25.5 & 24.8 \\
0 a 25 \% Trabajo & 30.0 & 29.0 & 28.0 & 27.0 \\
\hline
\end{tabular}

Fuente: ACGIH 2007, Guia 2 DS 055
- Temperatura de Globo (TG) vigila los efectos de radiación solar en la zona del infrarrojo directa sobre una superficie expuesta

- Temperatura del aire (TA), además de la humedad relativa (HR).

- Entrada / Salida de función muestra el valor con WBGT o sin exposición directa al sol

- Apagado automático con anulación

Capacidad de la interfaz RS-232 con Windows opcionales (B) software compatible.

\section{Resultados y discusión.}

Las mediciones efectuadas con el equipo EXTECH, fueron evaluados estadísticamente usando la diferencia de medias. En el cuadro 4 se muestra los valores obtenidos para las actividades de confitado caliente y tostado y secado, habiéndose medido las temperaturas a la altura de $1 \mathrm{~m}$ durante 1 hora desde las 11 a.m. hasta las 12 a.m. en intervalos de 15 minutos cada uno durante el día 26 de febrero del 2015; los datos obtenidos fueron promediados, de acuerdo con la norma técnica peruana (NTP) RM 375 -2008 En la tabla 4 se muestra los valores del índice WBGTi en ${ }^{\circ} \mathrm{C}$. 


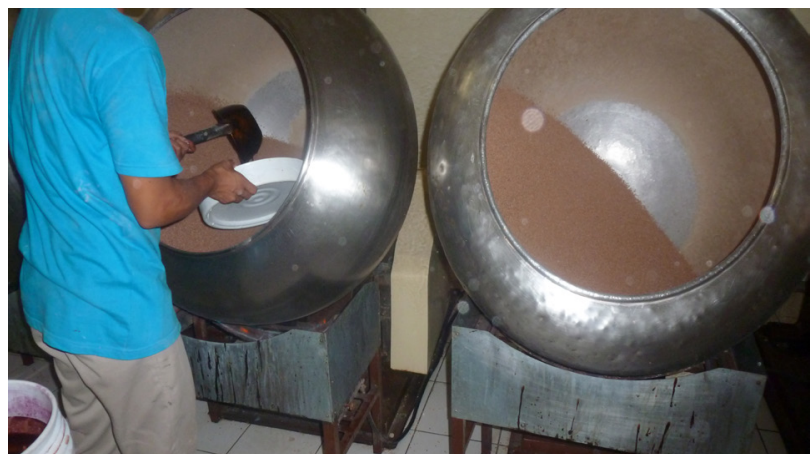

Los valores mostrados del Índice WBGTi mostrados en las tablas 4 y 5 fueron calculados con la relación 1 , para mediciones de estrés térmico en interiores.

Con respecto al gasto metabólico se estimó entre 125 $174 \mathrm{~W} / \mathrm{m}^{2}$ para ambas tareas, que de acuerdo a la tabla 1 corresponde a la categoría de ligero tanto para la tarea de confitado caliente y tostado y secado encontrando la razón trabajo/descanso en $75 \% / 25 \%$ en condición de aclimatado.

Figura 1. Foto trabajador en el area de confitado caliente.

Tabla 4 . Valores medidos y calculados de las diferentes temperaturas y humedades.

\begin{tabular}{cccccc}
\hline Actividad & $\begin{array}{c}\text { Temp. Seco } \\
\left({ }^{\circ} \mathrm{C}\right)\end{array}$ & $\begin{array}{c}\text { Temp. Globo } \\
\left({ }^{\circ} \mathrm{C}\right)\end{array}$ & $\begin{array}{c}\text { Humedad } \\
(\%)\end{array}$ & $\begin{array}{c}\text { WBGTi } \\
\left({ }^{\circ} \mathrm{C}\right)\end{array}$ & $\begin{array}{c}\text { Gasto metabólico } \\
\left(\mathrm{W} / \mathrm{m}^{2}\right)\end{array}$ \\
\hline Confitado Caliente & 34.7 & 40.6 & 49.9 & 31.7 & 125 \\
Tostado y Secado & 39.7 & 45.6 & 40.1 & 32.9 & 174 \\
\hline Fuente: Elaboración propia & & & &
\end{tabular}

En la tabla 5 se muestra los valores de WBGTi medidos comparados con las normas del límite máximo permisible y los limites de acción. Encontrándose los valores $3.2{ }^{\circ} \mathrm{C}$ y $4.4{ }^{\circ} \mathrm{C}$ por encima del límite de acción y $1.2^{\circ} \mathrm{C}$ y $2.4{ }^{\circ} \mathrm{C}$ mayor que el límite máximo permisible (LMP).

\section{Conclusiones}

Los índices de WBGTi oscilan entre 31.7 y $32.9{ }^{\circ} \mathrm{C}$ para las zonas; Confitado Caliente y Tostado - Secado respectivamente.

Los valores del índice WBGTi están por encima de los niveles de límite de acción en 3.2 y $4.4^{\circ} \mathrm{C}$ respectivamente.

Los valores del índice WBGTi están por encima de los niveles del límite máximo permisible en 1.2 y $2.4{ }^{\circ} \mathrm{C}$, respectivamente.

Los valores del gasto metabólico para las actividades evaluadas están entre 125 - $174 \mathrm{~W} / \mathrm{m}^{2}$ valores para actividades de nivel ligero.

\section{Recomendaciones}

Estudiar con más detalle la rutina de los trabajadores en los dos procesos a fin de establecer una mejor norma de trabajo (protocolo), cuidando el bienestar de los trabajadores.

Analizar la colocación de los extractores a fin de dar un flujo de enfriamiento más homogéneo en los ambientes de trabajo.

Realizar por lo menos anualmente una revisión médica de los trabajadores de las aéreas de trabajo.

\section{Literatura citada}

ACGIH (The Association Advancing Occupational and Environmental Health, US), 2001. Documentation of the Threshold Limit Values for. Physical Agents 7th Edition.

DS-055-2010 (Decreto Supremo, Perú), MEM 2010. (Ministerio de Energía y Minas), Reglamento de Seguridad y Salud Ocupacional y otras medidas complementarias en Minería.

DS 42F, 1964. (Decreto Supremo Peru), El Reglamento De Seguridad Industrial.

Iñaki A, Ergo K. 2000?. Aproximación al Riesgo y Confort Térmico en el trabajo. (en línea). España. Consultado 20 set. 2015. Disponible en www. ergokprevencion .org/Organizador/Doc/Frio $\% 20 \mathrm{~K} \% 20$ V01.pdf.

NTP 18 (Norma Técnica de Prevención, España). (Estrés Térmico. Evaluación de las exposiciones muy intensas.

NTP (Norma Técnica Peruana), RM 375 -2008 (Resolución Ministerial, sector Ministerio de Trabajo y Promoción del Empleo), Norma Básica de Ergonomía y de Procedimiento de Riesgo Disergonómico. 
RM 318-2010-MEM/DM (Resolución Ministerial

Peruana sector Energía y Minas), Modifican el

Reglamento de Seguridad y Salud en el Trabajo de las Actividades Eléctricas. 